\title{
Qualitative and Quantitative Evaluation of Charge Capture by a Radiology Information System
}

\author{
David R. Pickens, James A. Patton, and S. Julian Gibbs
}

\begin{abstract}
Installation of a radiology information management system (RIS) is usually justified on the basis of improved departmental efficiency and improved charge capture. However, evaluation of the success of these expected improvements is often difficult. The installation and operation of such a system in a medium-sized tertiary care hospital has permitted the effects of the RIS on the operation of the department to be studied and the improvements in charge capture provided by the system to be quantitatively assessed. As a result of a side-by-side comparison with a conventional check-sheet manual billing system, it is apparent that the RIS reduces the errors inherent in manual systems. Subjectively, it is also apparent that personnel prefer the computerized system to the manual charge sheets.

(c) 1990 by W.B. Saunders Company.
\end{abstract}

KEY WORDS: Management systems, information management, radiology information system, charge capture, billing, computers.

$\mathbf{M}$ AINTAINING RECORDS of charges in a radiology department that performs 140,000 to 160,000 studies a year is a substantial bookkeeping task. The usual method of recording charge data related to the examination is the use of charge sheets that the technologist performing the radiologic study marks or checks to indicate the procedure performed and materials used. The charge sheet permits the technologist to record the events rapidly and accurately on a sheet that has the patient's hospital number stamped on it and is preprinted with all the studies and materials likely to be used.

At the end of each working day, the collection of charge sheets is submitted to data processing personnel, who transcribe the marked procedure from each sheet into a small computer that creates a billing tape suitable for submission to the computerized charge-posting system. To maintain a proper record of these charges, the department keeps a copy of the original sheet and a written $\log$ of each procedure. The time required to post a charge to a patient's account from a manual entry system is usually 2 days; the charge is processed during the day after the service and posted in the early hours of the following morning.
This type of manual record-keeping system can function very well if personnel are careful and if records are reviewed to ensure that all the appropriate sheets are passed to the data processing department for data entry. However, there are several types of errors that can cause delayed or incorrect charge posting or lost charges. Delayed charges usually occur when a technologist is very busy. The charge sheets can be left in the radiographic area until they are discovered at a later time, whereupon they are turned in and posted late. Even if the technologists are very conscientious about processing these sheets, invariably there will be delays.

The second problem, incorrect charges, is caused by marking the wrong charge on the charge sheet or by data processing errors in which the correct charge is recorded by the technologist but the wrong charge is posted. Sometimes additional marks on the charge sheets are interpreted by the data processing personnel as valid charges. Incorrect charges due to spurious marks can sometimes be avoided if the technologist or other departmental personnel review a charge sheet before its submission to the data processing department.

Lost charges, the third problem, usually cannot be detected and properly billed because they do not exist as far as the billing process is concerned. These charges include those that are marked on a charge sheet that is lost or destroyed before it is sent to the data processing department and those that are never recorded by the technologist at all. Unrecorded charges often include those for materials and drugs used in complicated angiographic cases or in one of several procedures performed on a trauma patient. Even in a well-run facility, it is possible to lose some charges that should be billed. These

From the Vanderbilt University Medical Center, Nashville, $T N$.

Address reprint requests to David $R$. Pickens, PhD, Department of Radiology and Radiological Sciences, Vanderbilt University Medical Center, Nashville, TN 37232.

(C) 1990 by W.B. Saunders Company.

0897/90/0301-0011\$03.00/0 
are never recovered and are typically never detected, so no specific action can be taken.

The authors' department elected to install a radiology information management system (RIS) because the volume of studies was becoming increasingly difficult to process effectively with billing procedures that had undergone considerable refinement over several years. A number of benefits were expected from this installation, including increased efficiency of managing the department and improved charge capture, even though the manual system was considered to be nearly "airtight."

The RIS assigns an accession or sequence number to each study when the examination is scheduled. When the patient arrives in the department, a bar code is produced on a tracking form that indicates the patient name, hospital number, date of birth, examination to be performed, and other information. When the examination is finished, the technologist who performed the study uses a bar-code reader to indicate this to the RIS for the specified patient using the tracking card. At that point the RIS "knows" that the examination originally scheduled has been performed. If no corrections are made before closing the billing cycle, a billable charge is generated at midnight by the system, with no need for further interaction by departmental personnel. For those situations in which the examination is not performed, a report of scheduled but not completed examinations is produced. The personnel in the radiology office determine the reasons for uncompleted examinations and verify that all examinations are correctly recorded in the system.

\section{MATERIALS AND METHODS}

Before the RIS was used for billing purposes, it was decided that a comparison between the manual charge-sheet system and the RIS billing system should be made, for two reasons. The first was the reluctance of the financial management department to accept the computerized billing system without a comprehensive test. The second was the billing system of the RIS, which had been altered to accommodate the special needs of the study institution. Because this alteration changed the standard functioning of the RIS software, it was agreed that a series of tests would be performed before the billing tapes created by the system for billing purposes were used. The goal of these tests was to verify to everyone's satisfaction that the RIS would correctly capture all the charges for a patient's examination and produce a correct entry on the billing tape.

The best way to test the system was determined to be the performance of direct side-by-side comparisons of charge- sheet and logbook records with the records from the RIS This approach was implemented by accumulating all the charge sheets for the several days of each test and the billing reports from the RIS corresponding to each test day. Then, each charge sheet was compared with the record on the RIS. On a typical day during the week, this represented as many as 500 transactions; therefore, performing the comparisons required several hours each day. Weekends usually produced $40 \%$ to $50 \%$ of the average daily total of procedures. In those cases in which a charge in the RIS could not be verified by locating the charge in the manual reports, the film room was asked to pull the film folder for the patient to verify or disprove the entry.

\section{RESULTS}

\section{Daily Comparison}

The results available for a representative weekday during the 5-day test are typical of the results found daily in both test periods during the study. For the day that was studied, 536 transactions were recorded on the billing tape produced by the RIS. Five hundred transactions were recorded on the tape produced by the data processing personnel from charge-sheet information. Of these charges, 413 were recorded on the billing tape from the RIS and the tape produced from the charge sheets. Thus, 123 transactions were present only on the tape from the RIS, whereas 87 transactions were present only on the tape from the charge sheets.

Another 3-day comparison, including the Sunday of a holiday weekend and the following Monday and Tuesday, was performed. In this evaluation, a detailed comparison was made between the data on the billing tape report from the RIS and the transaction report reflecting transactions posted to the billing system. The examinations for Monday were compared one to one with the charges reported from the manual billing system for that day. Additionally, the following 4 days' reports from the manual system were searched for charges listed for Monday by the RIS but not found in the charge sheet report for Monday. This check was made to determine if some charges were posted on different dates by the manual system. For this day there were 73 examinations in question; they were investigated to determine why they did not appear on both billing lists.

Of the 73 examinations in question, 38 were found to be completed in the RIS system but were not posted to the billing system from the manual charge sheets. Of these, 9 examinations 
were correctly listed by the RIS system, 8 were listed by the RIS with a different date from the manual billing system, 9 were not reported in the manual system but films existed in the film file, 11 did not have a film file that could be located, and 1 emergency service charge could not be verified.

A second group of 9 examinations was posted from charge sheets, but they were not the examinations scheduled in the RIS. The RIS was correct for 8 of the examinations, and l examination was incorrect in both the RIS and in the posted charge.

A third category consisted of 26 examinations that were posted on the billing system but were not on the RIS examination list. Of these, 9 were completed the next day in the RIS, 8 were scheduled but not completed, 2 were processed and posted but never appeared in the RIS, 2 were partially correct in the RIS listing, and 5 could not be located.

In summary, Table 1 shows that the RIS was correct in 53 of the 73 examinations in question during this comparison. The RIS was correct outright in 36 exams. Nine were correct according to the films, but no report had been dictated. Eight were correct, but the technologists had not completed the examination to capture the charge. In only 3 of the 73 charges that were in question, the paper records were correct and the RIS was wrong. However, 1 charge could not be determined and 16 film file records could not be found. All the other records in the RIS were correct.

A significant problem that was found during the comparison was discrepancies in the date of service for a particular examination between the two billing systems. Once it was recognized that synchronization was impossible, the operational problems of incomplete examinations were addressed by revised procedures. These procedures used reports provided by the RIS to verify that

Table 1. Resolution of Examinations in Question

$\begin{array}{lr}\text { RIS correct } & 19 \\ \text { Different dates of service } & 17 \\ \text { Reports not done but films in film file } & 9 \\ \text { Scheduled but not completed } & 8 \\ \text { Could not locate films } & 16 \\ \text { Paper trail correct } & 2 \\ \text { Paper and RIS incorrect } & 1 \\ \text { Unsure (emergency examination) } & 1 \\ \text { Total no. examinations } & 73\end{array}$

scheduled examinations were completed and that unscheduled examinations were otherwise accounted for. At this point, the RIS chargecapture system was placed in production and the paper charge sheet system was eliminated.

\section{Long-Term Evaluation}

Because there was considerable interest by departmental administration in the performance of the RIS, a comparison was made between the billing results for 7 months before implementation of the RIS charge-capture system to 7 months after implementation of the RIS chargecapture system. The month during which the conversion from manual to RIS billing occurred was not used in the comparison. Total departmental revenue actually received was normalized by dividing each monthly figure by the total departmental revenue as budgeted for each month in the comparison period. This normalization helps to account for seasonal variations and price changes that could skew the data. The mean normalized total revenue, expressed as a percent, was found to be $114.1 \% \pm 6.2 \%$ for the 7 months before conversion to computerized charge capture. The average normalized revenue for the 7 months following the conversion to RIS charge capture was $115.1 \% \pm 4.5 \%$. This is a $1 \%$ increase for the period. However, this increase was found not to be statistically significant. Figure 1 shows the normalized data used in the comparison.

\section{DISCUSSION}

Several interesting discoveries were made during the comparisons of the charge sheets with the RIS charge-capture reports. The first was the realization that charges from the paper charge sheets would never match the charges recorded by the RIS on a day-to-day basis. This is true because the RIS is much more effective at recording the completion of an examination and reporting that information to the posting system than the manual charge sheets are. Using the RIS enables the radiology department to post charges in the early morning after the day of service, gaining one full day in the posting cycle. Most errors are corrected by office personnel, who verify that the examination in the RIS is the examination that was performed. Those errors that unavoidably go to posting are corrected by issuing a credit through the RIS. Because an 
Percent over budget

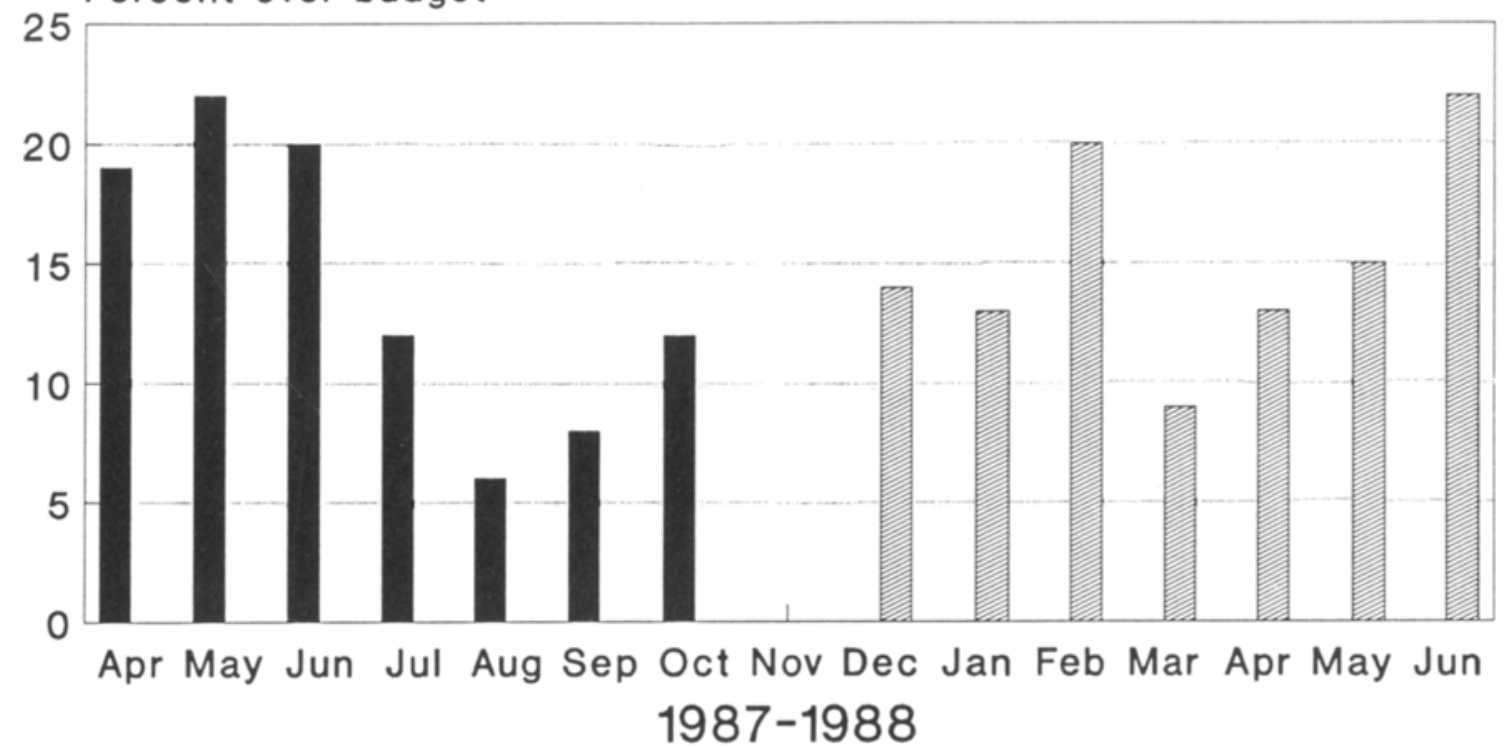

Series 1 एा: Series 2

Fig 1. RIS billing performance before and after conversion from manual charge sheets. Series 1,7 months before conversion; Series 2, 7 months after conversion. November 1987 was not included because conversion from charge sheets to computerized billing occurred during this time.

extra day has been gained in the billing cycle, these corrections usually occur before a bill is produced and sent to the patient.

The second discovery was that there seemed to be some significant inaccuracies in the manual system, primarily because of several people having to interact with the information before its being posted. Thus, there were additional opportunities for an error to be made on a patient's bill or a charge to be lost. Because the RIS eliminated many of the steps required to post the charge and provided additional reports of outstanding charges, it enabled the office personnel to ensure with a much higher degree of accuracy that the charges that were posted were correct.

In addition to providing the department with greatly enhanced capability for billing the correct charges, the elimination of most of the paper logbooks and schedule sheets has greatly increased the efficiency of the radiology office. Before the installation of the system, the numbers of hand-written log entries and forms that were processed by office workers was substantial. An estimate of the average number of tasks per signed patient report produced by the department was made in a project proposal for installa- tion of a RIS. ${ }^{1}$ At that time, the average number of tasks performed manually by radiology department personnel was estimated to be 100 . Included are those tasks accomplished in the course of registering, verifying completion of the examination, collection of charge sheets, and logging of the charges. In the listing shown in Table 2, most of the activities are performed automatically in the course of scheduling and completing the examination through the RIS (indicated by asterisks).

From a purely subjective interpretation, no one would advocate returning to the days of paper charge sheets. The reactions from radiology office personnel and people in the billing office of the hospital indicate that return to the charge-sheet method of processing billing information would not be acceptable and would result in lost revenues. Initial skepticism about the ability of the RIS to perform as expected turned into enthusiasm once the system was fully operational.

Data comparing departmental billing over several months before and after installation of the RIS do not show a statistically significant difference between preinstallation and postinstallation 
Table 2. Tasks Related to Charge Capture

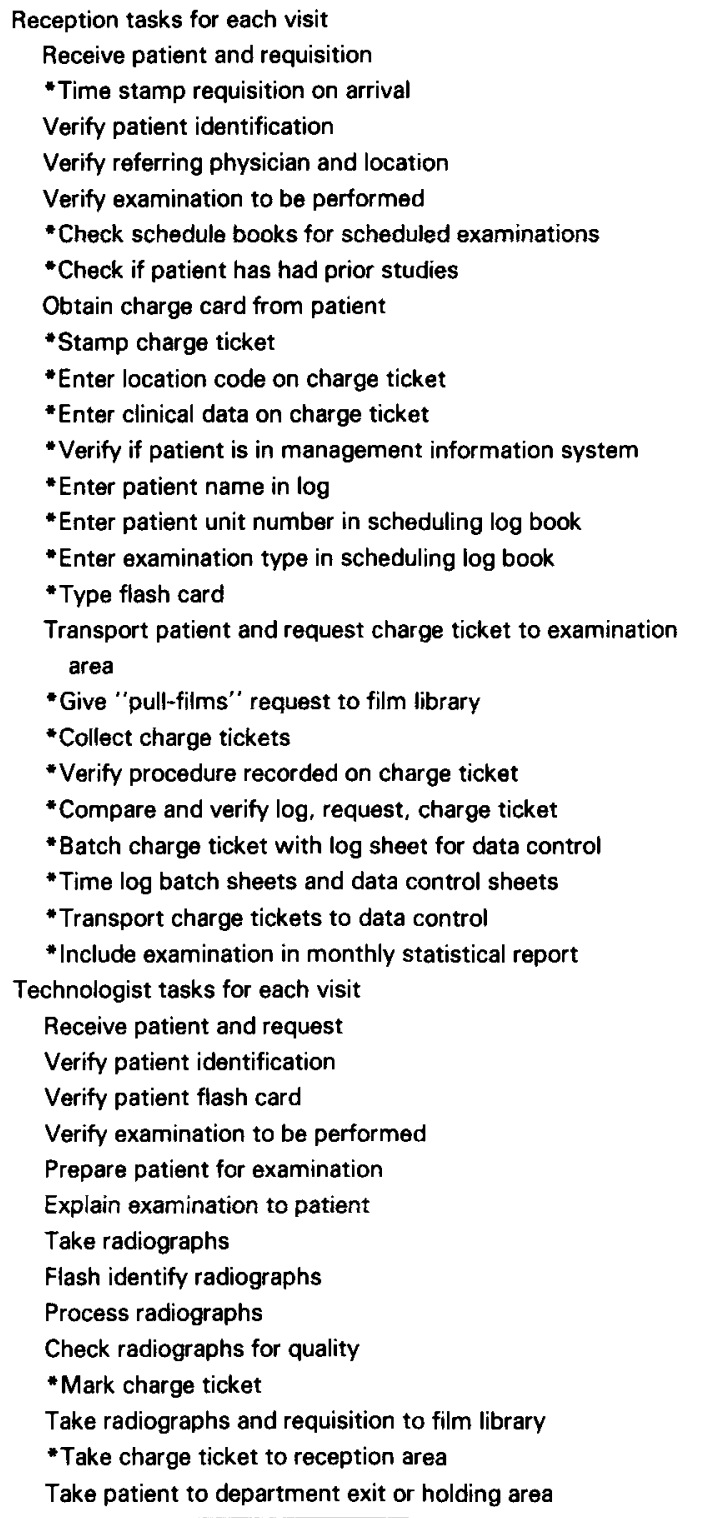

NOTE. Listed tasks are only part of the approximately 100 manual steps necessary to produce a report and bill the patient.

-Activities performed automatically by RIS. revenues. This is consistent with the substantial changes in revenue seen on a monthly basis due to price changes, seasonal variations, and changes in referral patterns, among others, that are not accounted for in the budgeting process. Even when revenue data are normalized by dividing by the budgeted amount for the month to obtain a percentage of the budget, fluctuations in revenue effectively mask the contribution of the RIS system to improved charge capture. If it is assumed that a good manual billing system is in place, a RIS replacing it might be expected to provide improvements in charge capture by $1 \%$ or $2 \%$. This sort of improvement cannot be seen in the data represented in Fig 1. That there is not a significant difference is an indicator that the manual system had worked relatively well.

Installation of a RIS is a major undertaking for a radiology department. However, it can be shown that the system will quickly increase the overall efficiency of the department and the ability of the users to process increasing numbers of studies. Furthermore, more quantitative investigation of the effect of the system on charge capture suggests that a properly selected and installed RIS can yield measurable benefits compared with a typical manual billing system.

A RIS offers many advantages to a radiology department when the system is matched to the environment. It improves employee effectiveness, lightens the loads imposed by manipulating large amounts of paper records, and reduces the incidence of errors in the department that can result in incorrect or missing charges. It can contribute to improvements in scheduling, registration, film management, and departmental operation that are difficult to quantitate; in general, enabling the department to provide better patient care. ${ }^{2,3}$

\section{REFERENCES}

1. Kaye JJ, Pickens DR, Hamilton R: Project evaluation report: Radiology management system. Nashville, TN: Vanderbilt University Medical Center, Department of Radiology and Radiological Sciences, August 1985 (internal document)
2. Jost RG: Monitoring department efficiency and quality, in Arenson RL (ed): Use of Computers in Radiology. Radiol Clin North Am 24:27-36, 1986

3. Bauman RA: The registration and scheduling process, in Arenson RL (ed). Radiol Clin North Am 24:5-9, 1986 